\title{
Die endoskopische Therapie der Tumorobstruktion des Tracheobronchialsystems: eine Gegenüberstellung verfügbarer Techniken
}

\author{
Endoscopic Treatment for Tumorous Obstructions of the Tracheobronchial \\ System: A Comparison of Available Techniques
}

Autoren

Institut
J. Hetzel, M. Böckeler

Medizinische Klinik, Abteilung II, Hämatologie, Onkologie, Immunologie, Rheumatologie und Pulmologie, Tübingen

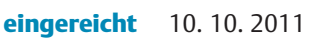
akzeptiert nach Revision 2. 1.2012

Bibliografie

DOI http://dx.doi.org/ 10.1055/s-0031-1291647 Online-Publikation: 24.5.2012 Pneumologie 2012; 66: 408-415 (c) Georg Thieme Verlag KG Stuttgart · New York ISSN 0934-8387

Korrespondenzadresse Dr. med. Jürgen Hetzel Medizinische Klinik II Universitätsklinik Tübingen Otfried-Müller-Straße 10 72076 Tübingen juergen.hetzel@med.unituebingen.de

\section{Zusammenfassung \\ $\nabla$}

Die zentrale Atemwegsstenose ist eine häufige Komplikation bei Tumorerkrankungen der Lunge. Symptomatische Stenosen können endoskopisch häufig erfolgreich beseitigt werden. Hierzu stehen mehrere Verfahren mit unterschiedlichen Therapieansätzen zur Verfügung. Die Behandlung exophytischer Tumorstenosen zielt darauf ab, das in das Lumen ragende Tumorgewebe zu entfernen. Dabei sind Rekanalisationsverfahren mit Soforteffekt (mechanische Abtragung, Elektrochirurgie, Argon-Plasma-Koagulation, Laser, Kryoextraktion) von Techniken mit verzögerter Wirkung (Kryotherapie, Brachytherapie, photodynamische Therapie) zu unterscheiden. Stenosen, die durch Kompression von außen entstehen, können durch die Implantation von Silikonstents oder selbstexpandierenden Metallstents wiedereröffnet werden.

\section{Einleitung \\ $\nabla$}

Maligne Lungentumoren sind die am häufigsten neu diagnostizierten Tumorerkrankungen weltweit [1]. Bei Männern sind sie die häufigste, bei Frauen die zweithäufigste Todesursache durch maligne Erkrankungen. Das 5-Jahres-Gesamtüberleben liegt bei 16\% [2]. Der Großteil der Lungenkrebsfälle wird erst in einem lokal fortgeschrittenen oder metastasierten und damit in einem palliativen Stadium diagnostiziert. Insgesamt entwickeln 20-30\% der Patienten Komplikationen, die Folgen tracheobronchialen Tumorbefalls sind [3]. Neben dem Bronchialkarzinom können auch pulmonale Metastasen anderer fortgeschrittener Tumorerkrankungen zu einer Einengung der zentralen Atemwege führen. Die Folgen endobronchialer/-trachealer Stenosen können Husten, Atemnot bis hin zur respiratorischen Insuffizienz und Sekretverhalte mit der Entwicklung poststenotischer Infekte sein. Die endoskopi-

\section{Abstract \\ $\nabla$}

Malignant central airway obstruction is a common problem in lung cancer. A symptomatic stenosis can often be treated successfully using endoscopy. Different approaches for recanalisation are available. The goal of treating exophytic obstruction is to remove the endobronchial tumour growth. Mechanic debulking, electrocautery/diathermy, argon plasma coagulation, laser resection and cryoextraction are techniques that can provide immediate relief. Cryotherapy, brachytherapy and photodynamic therapy show delayed recanalisation effects. Silicone or self-expanding metallic airway stents can be used for the treatment of airway obstruction due to extrinsic disease to restore and maintain airway patency.

sche Behandlung solcher Stenosen kann zu Besserung der Symptome und der Lebensqualität führen [4].

Die Behandlung der malignen Stenose sollte grundsätzlich multimodal geplant werden und kann neben der interventionellen Endoskopie auch Chemotherapie, radioonkologische Maßnahmen und chirurgische Verfahren umfassen.

In dieser Zusammenfassung sollen die verschiedenen tracheobronchialen Rekanalisationsverfahren dargestellt und ihre Indikation aufgezeigt werden.

\section{Therapieverfahren}

$\nabla$

Die häufigsten Indikationen zur interventionellen Therapie zentraler tracheobronchialer Stenosen sind symptomatische Einengungen, Stenosen mit dem Risiko einer raschen Verschlechterung, Atelektasen mit verbliebener Perfusion und post- 


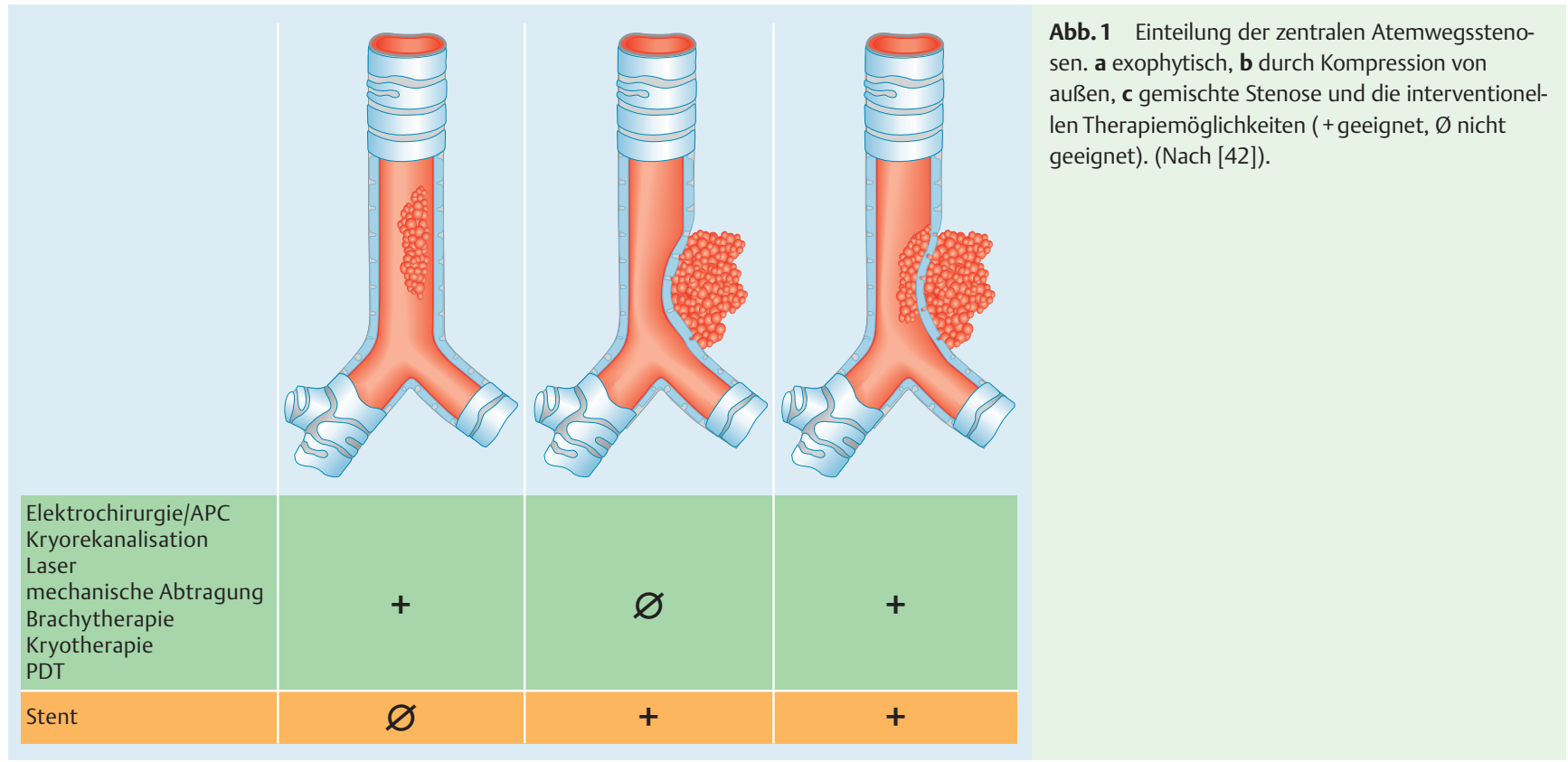

stenotische Pneumonien. Dabei kann die Stenose durch intraluminales Wachstum, durch extraluminales Wachstum mit Kompression von außen oder durch eine Kombination beider Faktoren entstehen ( $\mathbf{A b b} \mathbf{1}$ ). Eine Rekanalisation ist nur dann sinnvoll, wenn distal der Stenose Anschluss an das Bronchialsystem und an funktionsfähige Lungenabschnitte zu erwarten ist. Bei Stenosierungen durch Tumore, welche gut und sehr rasch auf eine Systemtherapie ansprechen (z. B. kleinzellige Bronchialkarzinome und hochmaligne Lymphome), kann durch den raschen Beginn einer Chemotherapie häufig auf eine Rekanalisationsmaßnahme verzichtet werden.

Die interventionelle Bronchoskopie ist in der Hand des erfahrenen Untersuchers häufig in flexibler Technik möglich. Dabei sollte jedem Patienten eine periinterventionelle Sedierung angeboten werden. Die Sedierung ermöglicht eine angstfreie Untersuchung und trägt zu optimalen und risikoarmen Untersuchungsbedingungen bei [5]. Je komplizierter eine Stenose und je risikoreicher die Intervention ist, desto wichtiger sind der sichere Atemwegszugang über einen flexiblen Tubus oder ein starres Bronchoskop und die Allgemeinanästhesie.

\section{Exophytische Tumorstenose}

Ist die Atemwegsstenose überwiegend durch exophytisches Tumorwachstum bedingt, so sind zur interventionellen Therapie Maßnahmen indiziert, die zur Abtragung des Tumorgewebes führen. Etablierte Verfahren hierfür sind mechanische Abtragungen, Kryoverfahren, Elektrochirurgie, Argon-Plasma-Koagulation (APC), Laser, Brachytherapie und die Photodynamische Therapie $(\mathrm{PDT})($ Abb.1).

\section{Mechanische Abtragung}

Hierunter werden die Möglichkeiten der Zangenabtragung zusammengefasst. Die mechanische Abtragung wird entweder allein oder in Kombination mit einer anderen, meist thermischen Technik angewendet. Die starre Bronchoskopie erlaubt im Vergleich zur flexiblen Bronchoskopie den Einsatz größerer Zangen, wodurch eine raschere Tumorabtragung möglich ist. Eine Sonderform der mechanischen Tumorentfernung stellt das „Coring out" dar. Hierbei wird im Rahmen einer starren Bronchoskopie
Tumorgewebe mit Hilfe des starren Rohres ausgeschält und anschließend mit der starren Zange entfernt.

\section{Kryotherapie/Kryoextraktion}

Die Kryoverfahren nutzen die Effekte von Kälte auf Tumorgewebe. Bei der bronchoskopischen Anwendung wird die Abkühlung der Sondenspitze durch den Joule-Thompson-Effekt hervorgerufen. Dieser besagt, dass die rasche Dekompression bestimmter Gase zur Energieaufnahme aus der Umgebung und damit zur Abkühlung führt. Dabei können innerhalb einer Sekunde lokale Temperaturen bis minus $89^{\circ} \mathrm{C}$ erreicht werden. In der Bronchoskopie kommen zumeist Kohlendioxid und Lachgas zum Einsatz [6].

Zwischen Kryotherapie und Kryoextraktion muss generell unterschieden werden. Ersteres Verfahren zielt darauf ab, Tumorgewebe lokal zu zerstören [7]. Dabei wird die Sondenspitze dem Tumor aufgesetzt. Durch wiederholte Gefrier- und Auftauzyklen werden dann im Tumorgewebe Nekrosen induziert. Das Tumorgewebe verbleibt jedoch im Bronchialsystem und muss daher häufig in einer Folgebronchoskopie entfernt werden. Die Kryotherapie ist ein sicheres Verfahren mit wenigen Komplikationen [6,8].

Die Kryoextraktion stellt dagegen eine Technik zur sofortigen Therapie einer Atemwegsstenose dar (Kryorekanalisation) [9, 10]. Zusätzlich eignet sich die Kryoextraktion zur Gewinnung von Gewebeproben für histologische Untersuchungen und wird dann als Kryobiopsie bezeichnet [11,12]. Neben der Extraktion von exophytischem Tumorgewebe können mit der Kryosonde auch wasserhaltige Fremdkörper und Blutkoagel aus dem Bronchialsystem entfernt werden [6].

Bei der Kryoextraktion wird die Sondenspitze dem Tumor aufgesetzt oder in den Tumor geschoben. Der Gefriervorgang führt zum Anhaften des Gewebes an der Sondenspitze. Durch Zug an der Kryosonde wird das Tumorgewebe gelöst und in einer kontinuierlichen Bewegung gemeinsam mit dem Bronchoskop aus dem Bronchialsystem entfernt ( Abb.2). Anschließend wird das Gewebe im warmen Wasserbad von der Sondenspitze abgelöst [9]. Da das Bronchoskop aus dem Bronchialsystem entfernt werden muss, sollte der Atemweg durch ein starres Bronchoskop oder einen flexiblen Tubus gesichert werden. 


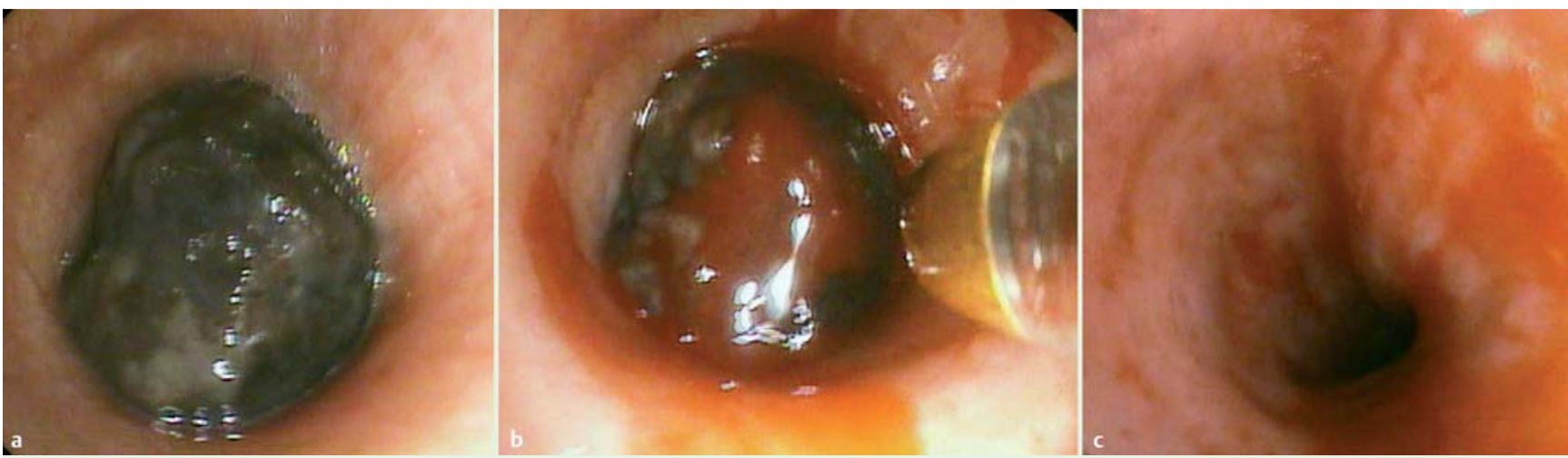

Abb.2 Kryoextraktion einer endobronchialen Melanommetastase. a vor Extraktion, b sichtbare Kryosondenspitze, c nach Extraktion.

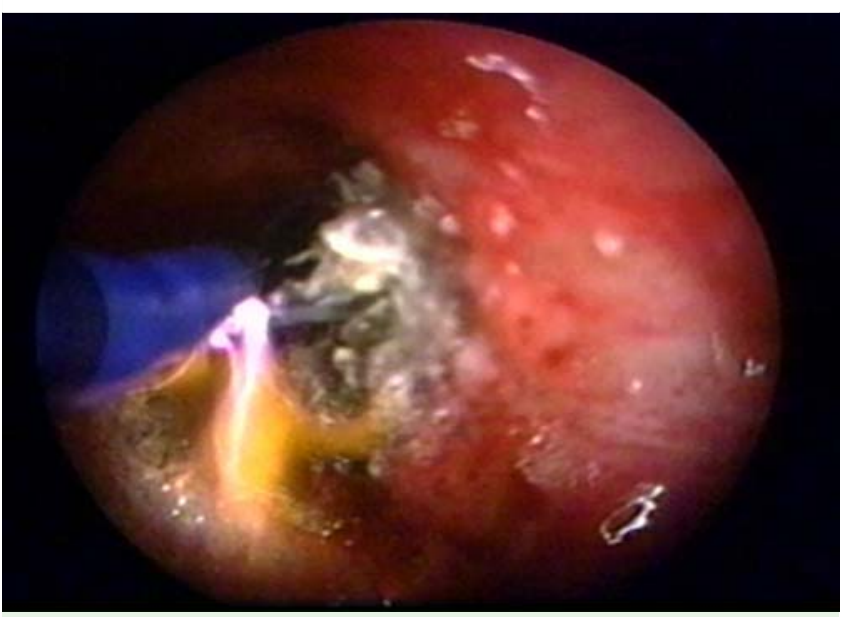

Abb. 3 Endobronchiale APC-Anwendung (freundlicherweise zur Verfügung gestellt durch Dr. G. Reichle).

\section{Elektrochirurgie (HF-Chirurgie, Elektrokauterisation)}

Argon-Plasma-Koagulation (APC)

Elektrische Ströme, die den Körper durchfließen, erzeugen nach dem Jouleschen Gesetz eine Erwärmung. Diese physikalische Eigenschaft macht man sich in der Elektrochirurgie und der APC zunutze, um Tumorgewebe zu zerstören. Mit der Elektrochirurgie und der APC ist es möglich, das Gewebe zu schneiden (Elektrochirurgie), zu koagulieren, zu karbonisieren und zu vaporisieren [13].

Die Elektrochirurgie benötigt für den Stromfluss direkten Kontakt zwischen Sonde und Gewebe. Eine Vielzahl verschiedener Sonden (Kugelsonden, Messer, Zangen, Schlingen usw.) ermöglicht es, die Energie an der gewünschte Stelle des Bronchialsystems zu applizieren. Damit sind jedoch teilweise unerwünschte Wirkungen, wie das Festkleben am Gewebe, verbunden $[14,15]$. Die APC stellt eine Weiterentwicklung der Elektrochirurgie dar. Bei dieser Methode ist die Gewebezerstörung ohne Kontakt möglich $[15,16]$. Der Stromfluss erfolgt über das Edelgas Argon, das über eine katheterförmige Sonde in das Bronchialsystem geleitet wird und unter hoher Wechselspannung ionisiert. Je nach Tumorlokalisation kann die Ausströmöffnung für das Argon axial, lateral oder radial ausgeformt sein. Die Eindringtiefe ist im Wesentlichen leistungsabhängig und liegt maximal bei $5 \mathrm{~mm}$ [17]. Sie wird durch die Denaturierung des Gewebes und der damit zusammenhängenden Widerstandserhöhung begrenzt [15]. Aufgrund ihrer sprayartigen, oberflächlichen Wirkung eignet sich die APC neben der Gewebezerstörung auch sehr gut zur Blutstillung ( $\bullet$ Abb.3).

Für die Therapie der exophytischen Tumorstenose werden zwei unterschiedliche Verfahren beschrieben. Am häufigsten erfolgt eine primäre Denaturierung des Tumors mit Elektrochirurgie bzw. APC, dann erfolgt die Abtragung der nekrotischen Reste mit der Zange oder der Kryosonde [15], ( $\bullet$ Abb.4). Alternativ kann der Tumor primär mechanisch oder mit der Kryosonde abgetragen werden, um dann, falls erforderlich, die Blutstillung mit APC durchzuführen [15].

Laser

Die Verwendung des Lasers in der Endoskopie begann Ende der 1970er-Jahre mit der Einführung des Nd-YAG-Lasers [18-20]. Die Wirkung des Lasers beruht auf der Erhitzung des Gewebes durch gebündeltes Licht. Die Energieaufnahme des Gewebes ist von der Leistung des Lasers, der Wellenlänge und den Gewebeeigenschaften wie z.B. der Gewebefarbe abhängig [19]. Dabei wird die höchste Energieaufnahme unterhalb der Gewebeoberfläche erreicht. In der Bronchoskopie hat der Nd-YAG-Laser die weiteste Verbreitung erfahren, da er ausreichende Leistung zur Vaporisation mit guten Koagulationseigenschaften kombiniert [15]. Aufgrund der präzisen und kleinflächigen Energieabgabe kann mit dem Laser sehr genau gearbeitet werden. Daher eignet sich der Laser auch zum Einschneiden membranartiger Stenosen $[19,20]$. Exophytische Tumore werden zumeist mit dem Laser koaguliert oder karbonisiert, um dann in einem zweiten Schritt mechanisch abgetragen zu werden [19]. Obgleich von manchen Autoren in flexibler Bronchoskopie gute Ergebnisse erreicht werden konnten [19,21], wird von den internationalen Fachgesellschaften die Durchführung der Lasertherapie in starrer Bronchoskopie empfohlen [22].

\section{Brachytherapie}

Die endobronchiale Brachytherapie verwendet ionisierende Strahlung zur Zerstörung von Tumorgewebe. Nach endoskopischem Einlegen eines Führungsdrahtes wird ein Katheter im Bereich des Tumors platziert. Durch diesen Katheter wird dann in Afterloading-Technik die Strahlenquelle (zumeist Iridium-192) eingeführt und in der Nähe des Tumors platziert. Dieses Verfahren ermöglicht die Applikation einer hohen Strahlendosis in dem um die Strahlenquelle liegenden Gewebe bei gleichzeitiger Schonung der extrabronchialen Strukturen [6]. 

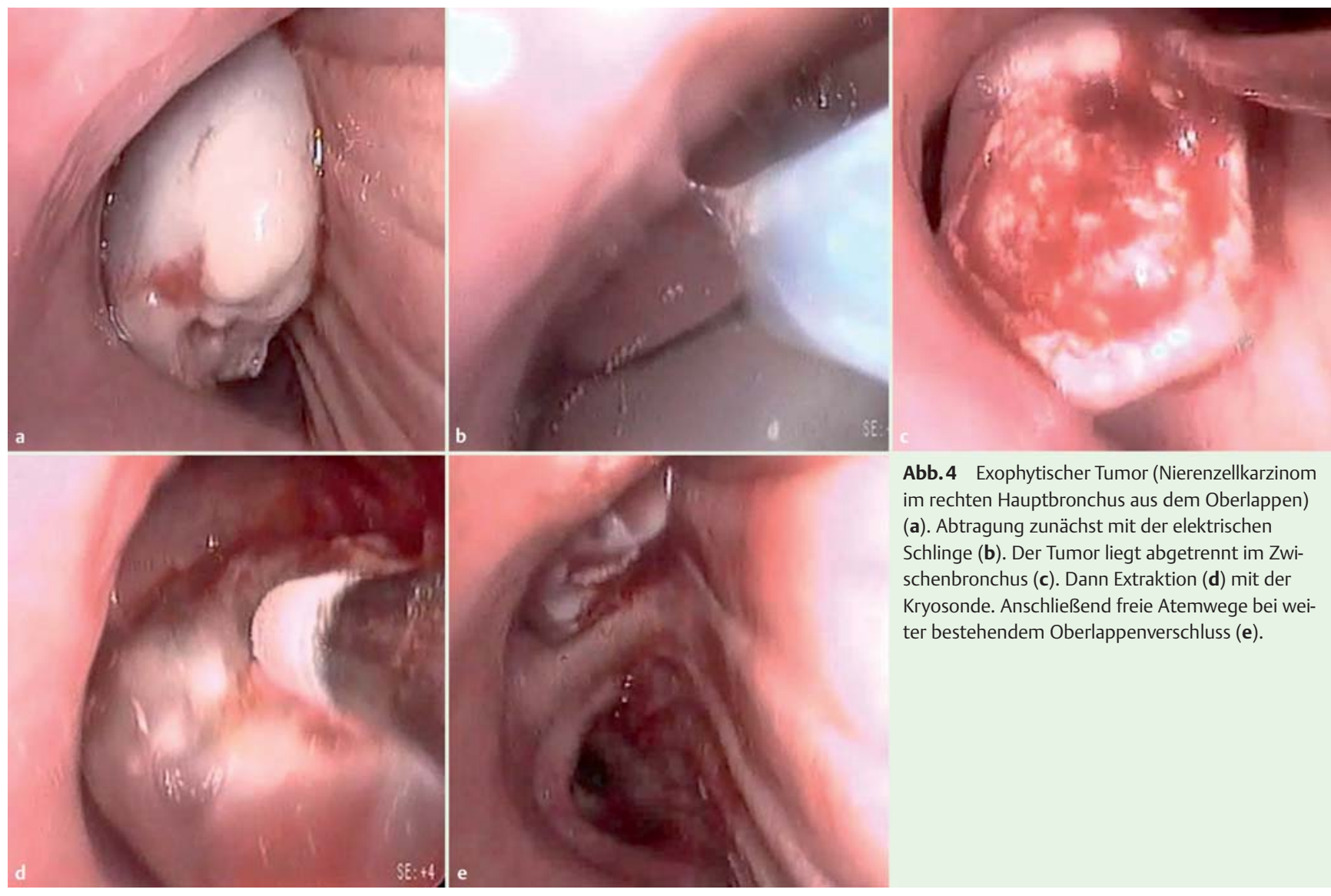

Abb.4 Exophytischer Tumor (Nierenzellkarzinom im rechten Hauptbronchus aus dem Oberlappen) (a). Abtragung zunächst mit der elektrischen Schlinge (b). Der Tumor liegt abgetrennt im Zwischenbronchus (c). Dann Extraktion (d) mit der Kryosonde. Anschließend freie Atemwege bei weiter bestehendem Oberlappenverschluss (e).

Photodynamische Therapie (PDT)

Die Photodynamische Therapie nutzt zwei Effekte aus: erstens die weitgehend selektive Anreicherung eines Photosensibilisators im Tumorgewebe und zweitens die Zerstörung dieses Gewebes durch Beleuchtung mit einem Licht definierter Wellenlänge. Der am häufigsten in der Bronchoskopie angewendete Photosensibilisator ist das Porphyrinderivat Photofrin, welches 2-3 Tage vor der Bestrahlung intravenös appliziert wird [23]. Die lichtinduzierte Aktivierung des Photosensibilisators führt in der Gegenwart von Sauerstoff zur Gewebezerstörung. Dieser Effekt tritt verzögert ein. Einige Tage nach der Lichtapplikation muss das nekrotische Gewebe abgetragen werden [6]. Während die PDT sowohl beim kleinzelligen als auch beim nicht-kleinzelligen Bronchialkarzinom effektiv ist $[6,23,24]$, können benigne Tumore aufgrund der fehlenden Anreicherung des Photosensibilisators nicht behandelt werden (zum Beispiel Granulationsgewebe). Die PDT ermöglicht sowohl die Behandlung von fortgeschrittenen exophytischen Malignomen wie auch die Behandlung von Frühkarzinomen. Aufgrund einer photofrinbedingten Photosensibilisierung der Haut müssen die Patienten Sonneneinstrahlung bzw. helles Licht bis zu 8 Wochen meiden [23,25]. Dies bedeutet eine erhebliche Einschränkung der Lebensqualität, weshalb die PDT in der palliativen Situation nur selten angewendet wird.

Vergleich der Verfahren bei exophytischer Tumorstenose Prospektive und randomisierte Untersuchungen zum Vergleich verschiedener Verfahren existieren bisher nicht. Bei der Bewertung der einzelnen Verfahren ist zu beachten, dass in den Publikationen zu den verschiedenen Verfahren jeweils eine entsprechende Patientenselektion erfolgte. Ein direkter objektiver Vergleich der Effektivität zwischen den verschiedenen Verfahren ist daher kaum möglich. Die differenzialtherapeutischen Strategien stützen sich daher auf die Daten der publizierten Studien sowie auf die technischen und biophysikalischen Eigenschaften des jeweiligen Rekanalisationsverfahrens. Die Auswahl des Rekanalisationsverfahrens basiert hauptsächlich auf folgenden Kriterien (৫ Abb. 5):

- Dringlichkeit der Stenosebeseitigung

- Erreichbarkeit des Tumors

- Komplikationsprofil der Rekanalisationsverfahren, Blutungsrisiko des Tumors

- Abhängigkeit von hoher inspiratorischer Sauerstoffkonzentration

- Aufwand und Kosten

- Persönliche Erfahrung

\section{Dringlichkeit der Stenosebeseitigung}

Die thermischen Verfahren, Laser, Elektrochirurgie und APC, sowie die Kryorekanalisation und die mechanische Abtragung erzielen einen Soforteffekt und eignen sich daher für symptomatische Atemwegsstenosen. Diese Verfahren erzielten in den verschiedenen Studien ähnliche Erfolgsraten (60-90\%) [6, 9,10]. Da die bei alleiniger Anwendung thermischer Verfahren erreichbare Gewebeschrumpfung bei großen exophytischen Tumoren zumeist keine ausreichende Wiedereröffnung der Atemwege ermöglicht, erfolgt die Kombination mit einem mechanischen Verfahren, um das koagulierte und denaturierte Gewebe aus dem Bronchialsystem zu entfernen (Zange oder Kryoextraktion, welche im Grunde genommen eine mechanische Rekanalisationstechnik darstellt). 
Die Brachytherapie, die Kryotherapie und die Photodynamische Therapie entfalten ihre Wirkung erst nach Tagen oder Wochen und sind daher bei Patienten, die einer raschen Rekanalisation bedürfen, nicht geeignet [6].

\section{Erreichbarkeit des Tumors}

Die Erreichbarkeit des exophytischen Tumorgewebes hängt im Wesentlichen von Tumorgröße, -form und -lokalisation sowie der Präzision, Flexibilität und Wirkrichtung der Rekanalisationstechnik ab. Methodenbedingt kann mit dem Laser und der Zange nur in axialer Richtung des Bronchoskops gearbeitet werden. Die APC, die Elektrochirurgie und die Kryoverfahren erlauben die Anwendung sowohl in axialer als auch in radiärer Richtung, während die Brachytherapie die Strahlung unselektiv radiär abgibt. Mit der Argon-Plasma-Koagulation können Läsionen, welche nicht in der direkten Verlängerung des Endoskops liegen, behandelt werden. Es kann „um die Ecke“ therapiert werden. Allerdings ist die Präzision nicht so hoch wie beim Laser, der Elektrochirurgie und den Kryoverfahren, was die Anwendbarkeit auf Lappenbronchus- und Segmentbronchusebene einschränkt. Der Laser kommt vorwiegend in der Trachea, den Hauptbronchien und im Zwischenbronchus zum Einsatz. Aufgrund der Flexiblität und der Wirkrichtung können die Kryoverfahren, die Brachytherapie und die photodynamische Therapie auch auf Lappenbronchus- und Segmentbronchusebene eingesetzt werden. Hierbei muss beachtet werden, dass die Indikation zur Rekanalisation in den Segmentbronchien jedoch nur sehr selten gegeben ist.

Neben den Eigenschaften der Rekanalisationstechnik wird die Erreichbarkeit auch durch Tumorgröße und Tumorform beeinflusst. Große exophytische Tumore können mit der Kryotherapie, der PDT und der Brachytherapie aufgrund der limitierten Wirktiefe nicht ausreichend behandelt werden. Diese Verfahren eignen sich hingegen für die Therapie des auf die Mukosa beschränkten Frühkarzinoms in kurativer Intention [5]. Die Abtragung großer exophytischer Tumore ist sowohl mit den thermischen Verfahren (zumeist in Kombination mit mechanischen Verfahren) als auch mit der mechanischen Abtragung und Kryoextraktion möglich. Neoplasien, welche polypartig in das Lumen ragen, eignen sich zur elektrochirurgischen Abtragung mit der Schlinge. Hierbei wird durch die Koagulation der Gefäße im Tumorstiel das Blutungsrisiko deutlich vermindert $[14,26]$.

\section{Komplikationen und Blutungsrisiko}

Stark vaskularisierte Tumore wie das Nierenzellkarzinom und das Karzinoid neigen bei Manipulation zu stärkeren Blutungen. Bei solchen Tumoren werden für die Akutrekanalisation thermische Verfahren bevorzugt, da diese aufgrund ihrer Koagulationseigenschaften die Gefäße veröden können. Dabei wird der hämostatische Effekt der APC höher eingeschätzt als die des Lasers [15]. Wenn die Kombination mit einem mechanischen Verfahren erforderlich ist, kann es jedoch zu einer Blutung aus dem Wundgrund kommen. Die mechanische Rekanalisation und die Kryorekanalisation können Blutungen verursachen, die jedoch in der Regel gut kontrollierbar sind [9,10]. Sämtliche Verfahren mit verzögertem Wirkungseintritt haben während der Anwendung ein geringeres Perforations- und Blutungsrisiko. Allerdings wurde bei der Brachytherapie im mittel- und längerfristigen Verlauf eine gehäufte Anzahl fataler Blutungen (ca. 10\%), Atemwegsstenosen (10\%) und die Ausbildung von Fisteln beschrieben [6]. Ein wichtiges Kriterium für die Auswahl des Rekanalisationsverfahrens ist die Kontrollierbarkeit, mit der Tumorgewebe zerstört wird. Je besser die Kontrolle, desto leichter können Schäden an

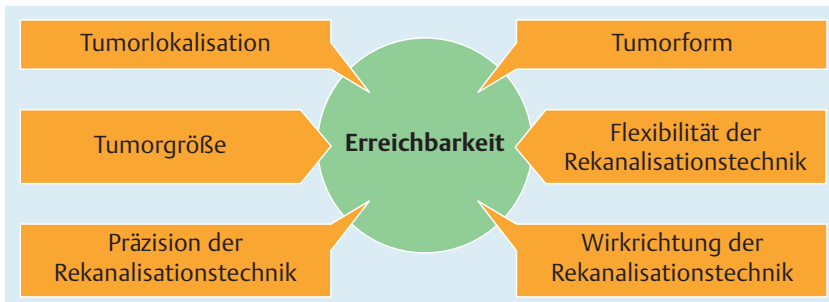

Abb. 5 Faktoren, die die Erreichbarkeit des Tumors beeinflussen.

gesunden Strukturen und Perforationen vermieden werden. Bei den Kryoverfahren, der Elektrochirurgie und der APC sind die Wirkung und deren Ausdehnung gut sichtbar. Im Vergleich zur APC erlauben jedoch die Kryoverfahren und die Elektrochirurgie eine präzisere Lokalisation, weshalb die Schädigung gesunder Bronchialschleimhaut seltener erfolgt. Die Laseranwendung ist in der Hand des Geübten gut kontrollierbar und präzise lokalisierbar. Da der hauptsächlich verwendete Nd-YAG-Laser eine Tiefenwirkung besitzt, die nicht einsehbar ist, besteht das Risiko einer Perforation [15]. Eine ähnliche Tiefenwirkung ist auch mit der Elektrochirurgie erzielbar, weshalb diese auch ein Perforationsrisiko birgt. Das Perforationsrisiko durch Kryoverfahren ist gering. Bei der Kryorekanalisation ist das Perforationsrisiko vor allem dann gegeben, wenn in Bereichen, die nicht durch Knorpel stabilisiert sind, Gewebe extrahiert wird (z.B. Tracheahinterwand) [10]. Bei der PDT und der Brachytherapie können die Effekte nicht direkt kontrolliert werden, das Perforationsrisiko ist jedoch gering.

Prinzipiell gilt: Je schlechter eine Leitstruktur bei der Rekanalisation erkennbar ist und je größer die Tiefenwirkung des Verfahrens ist, desto höher ist das Perforationsrisiko, desto wichtiger ist eine vorsichtige und präzise Vorgehensweise.

Sowohl bei der APC als auch bei der Anwendung des Lasers wurden klinisch relevante arterielle Gasembolien beschrieben [27, 28].

Die Effekte von Elektrochirurgie, APC und Laser beruhen auf Hitzeentwicklung. Es kann bei hohen inspiratorischen Sauerstoffkonzentrationen zu endobronchialen Bränden kommen [15]. Daher muss bei thermischen Verfahren die inspiratorische Sauerstoffkonzentration sicher auf $<35-40 \%$ begrenzt werden. Zudem birgt die Hitzeentwicklung das Risiko einer Bronchoskopschädigung. Es ist deshalb stets auf einen ausreichenden Abstand zwischen Applikationssonde und Endoskopspitze zu achten. Bei der Photodynamischen Therapie, der Brachytherapie und der mechanischen Abtragung bestehen keine temperaturbedingten Probleme. Kälteschäden am Bronchoskop durch die Kryoverfahren sind nicht bekannt.

\section{Aufwand}

Bei der Lasertherapie müssen spezielle Sicherheitsvorschriften wie z.B. die Brillenpflicht genau beachtet werden. Im Vergleich zur Brachytherapie mit ihrem hohen organisatorischen Aufwand - Koordination von Bronchoskopiker, Strahlentherapeut und evtl. Anästhesist - sind die mechanische Abtragung, die Kryoverfahren und die Elektrochirurgie/APC unkompliziert und rasch anwendbar. Ein weiterer Nachteil der Brachytherapie besteht darin, dass die Gesamtstrahlendosis fraktioniert in mehreren Sitzungen appliziert werden muss.

Bei allen thermischen Verfahren wie auch bei der PDT und der Kryotherapie kommt es in den Tagen nach der Anwendung zu Fibrinausschwitzungen und endobronchialen Nekrosen, welche 
Tab. 1 Therapeutische Indikationen bei malignen exophytischen Tumorstenosen.

\begin{tabular}{|c|c|c|c|c|c|c|c|}
\hline Indikation & $\begin{array}{l}\text { Mechanische } \\
\text { Abtragung }\end{array}$ & Laser & HF/APC & Kryoextraktion & Kryotherapie & PDT & Brachytherapie \\
\hline $\begin{array}{l}\text { Tumorstenose mit } \\
\text { akuter Dyspnoe }\end{array}$ & ++ & $++*$ & $++*$ & ++ & - & - & - \\
\hline $\begin{array}{l}\text { Tumorstenose ohne } \\
\text { akute Dyspnoe }\end{array}$ & ++ & $++*$ & $++^{*}$ & ++ & ++ & + & + \\
\hline distaler Tumor & + & + & + & ++ & ++ & ++ & ++ \\
\hline Koagulation & - & ++ sofort & ++ sofort & - & ++verzögert & - & ++verzögert \\
\hline
\end{tabular}

++ gut geeignet, + mäßig geeignet, - nicht geeignet; * bei großen exophytischen Tumoren häufig Kombination mit mechanischer Abtragung oder Kryoextraktion (modifiziert nach Vergnon [6]).

zu einer erneuten Einengung der Atemwege führen können. Daher ist es erforderlich, die Gewebenekrosen und Fibrinmembranen in Folgebronchoskopien abzutragen. Derartige Effekte treten bei der mechanischen Abtragung und der Kryorekanalisation nicht auf.

Die Bewertung der einzelnen Verfahren wird in $\bullet$ Tab. 1 zusammengefasst.

\section{Stenose durch Tumorkompression von außen}

Die tracheobronchiale Stenose durch eine Tumorkompression von außen ist die Hauptindikation zur Verwendung von Platzhaltern (Stents) [4]. Den ersten Bronchialstent legte von Trendelenburg chirurgisch ein [29]. Im Laufe der letzten Jahrzehnte wurde eine Vielzahl verschiedener Stents entwickelt, die sich folgenden Kategorien zuordnen lässt: Silikonstents und Metallstents ohne oder mit Kunststoffüberzug (Coverung).

Die entscheidenden Einflussfaktoren für die Stentwahl sind die Anatomie der Stenose, die Länge und der Durchmesser der Stenose [30]. Die Wahl des Stentdurchmessers ist entscheidend für den Therapieerfolg. Während bei zu kleinen Stents das Risiko der Migration hoch ist, kann es bei zu großen Stents zu vermehrter Granulationsgewebsbildung kommen [30]. Hu berichtet, dass bei Dumon-Stents das Risiko der Granulationsgewebsbildung erhöht ist, wenn das Verhältnis von Stentdurchmesser zu Atemwegsdurchmesser größer als 0,9 ist [31]. Der Stent sollte so platziert werden, dass der Stent nach der Implantation das proximale und das distale Ende der Stenose um ca. $5 \mathrm{~mm}$ überragt [32].

Bei Stenosierungen im Bereich der Hauptcarina kommen YStents zum Einsatz. Diese sind sowohl als Silikonstents (DumonY-Stent, Novatech, Frankreich) [33], Metallstents (z. B. ecostent Y Carina, Leufen medical, Deutschland) und als dynamische Hybridstents (Freitag-Stent, Rüsch, Deutschland) verfügbar [3]. Besteht eine Einengung im Bereich eines Tracheostomas, eignet sich der T-förmige Montgomery-Stent.

\section{Silikonstents}

Der am häufigsten verwendetet gerade Silikonstent ist der von Dumon eingeführte Stent mit außen liegenden Noppen [3, 34] ( Abb.6). Die Implantation wird in starrer Bronchoskopie durchgeführt. Vorteile der Dumon-Stents (Novatech, Frankreich) sind, dass Repositionierung, Stententfernung und Replatzierung gut möglich sind [3]. Die Stents sind in verschiedenen Längen und Durchmessern erhältlich. Um die optimale Stentlänge zu erreichen, können die Stents im Bronchoskopielabor direkt vor der Implantation auf die optimale Länge gekürzt werden. In der größten Untersuchung über Dumon-Stents wurde deren Wirksamkeit nachgewiesen [35]. Die häufigsten Komplikationen waren Migration (9,5\%), Granulationsgewebsbildung (7,9\%) und sekretbedingte Atemwegsobstruktion (3,6\%).

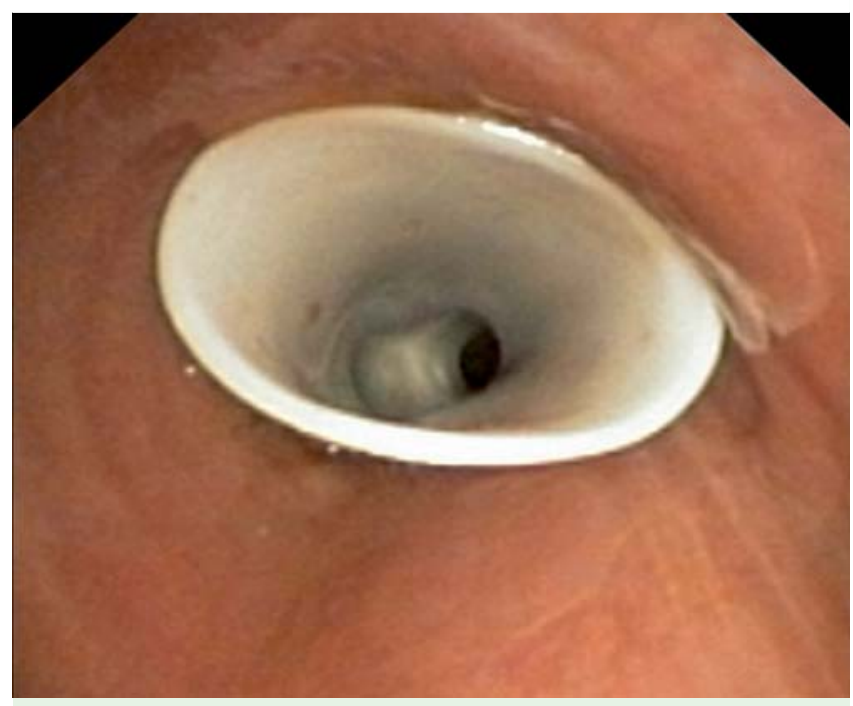

Abb. 6 Dumon-Y-Stent (freundlicherweise zur Verfügung gestellt von Prof. Dr. L. Freitag).

Die Entfernung von Silikonstents erfolgt in starrer Bronchoskopie und ist in der Regel gut möglich.

\section{Metallstents}

Tracheobronchiale Metallstents wurden von Gefäß- und Gallengangsstents abgeleitet [30,36]. Initial war zur Entfaltung dieser Stents eine Ballondilatation erforderlich. Heutzutage werden Metallstents aus einer Nickel-Titan-Legierung (Nitinol) hergestellt und bestehen aus selbstexpandierenden Drahtgeflechten ( $\bullet$ Abb. 7). Nitinol gehört zu den Werkstoffen, die ihre Form nach mechanischer Einwirkung wieder annehmen. Daher können die eng zusammengefalteten Nitinolstents im Gegensatz zu den Silikonstents in flexibler Technik eingeführt und in der Stenose expandiert werden. Die Implantation erfolgt in Seldingertechnik unter radiologischer oder bronchoskopischer Kontrolle [37]. Die Repositionierung ist schwieriger als bei den DumonStents. Eine Reimplantation in Seldingertechnik nach Entfernung ist konstruktionsbedingt bei manchen Stents (z.B. UltraflexStent ${ }^{\circledR}$, Boston Scientific, USA) nicht, bei anderen (z. B. aer-Stent ${ }^{\circledR}$, Leufen medical, Deutschland) nur schwer möglich.

Aufgrund der Struktur des Drahtgeflechtes besitzen moderne Nitinolstents eine hohe Flexibilität und ermöglichen eine gute Adaptation an die anatomischen Gegebenheiten, ohne dass eine longitudinale Verschiebung bei Kompression von außen auftritt $[30,38]$. Die im Vergleich zu den Dumon-Stents geringere Wandstärke der Metallstents ermöglicht ein größes Lumen bei gleichem Stentdurchmesser. Dieser Effekt ist in den Bronchien relevanter als in der Trachea. 


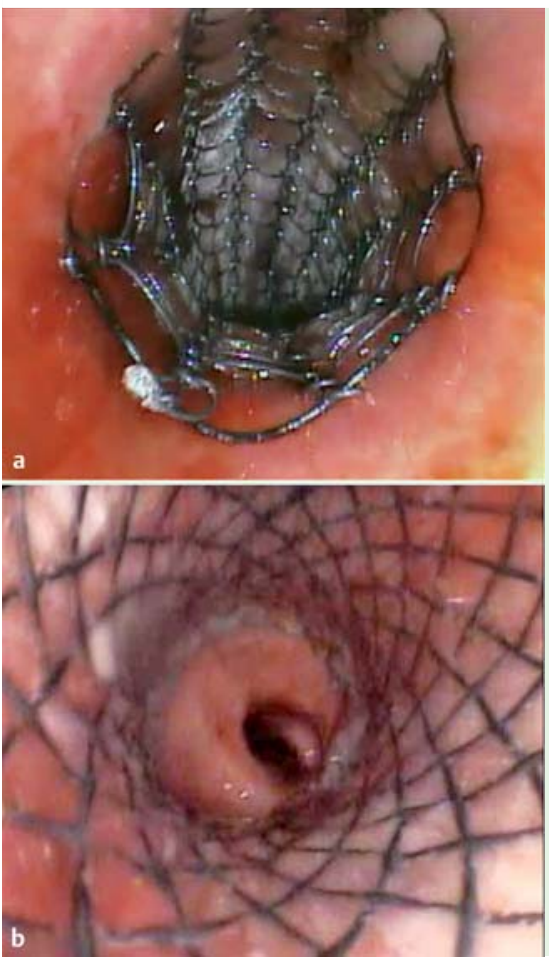

Abb.7 Metallstents. a Ultraflex-Stent ${ }^{\circledR}$ (Boston Scientific). b aer-Stent ${ }^{\circledR}$ (Leufen).

Tab. 2 Vor- und Nachteile von Silikon- und Metallstents (modifiziert nach Casal [19]).

\begin{tabular}{|c|c|}
\hline Silikon & Metall \\
\hline $\begin{array}{l}\text { starre Bronchokopie mit } \\
\text { Allgemeinanästhesie }\end{array}$ & flexible Bronchoskopie \\
\hline $\begin{array}{l}\text { schwerer einzulegen, leichter zu } \\
\text { entfernen }\end{array}$ & $\begin{array}{l}\text { leichter einzulegen, schwerer zu } \\
\text { entfernen }\end{array}$ \\
\hline häufiger Migration & seltener Migration \\
\hline $\begin{array}{l}\text { geringerer Innendurchmesser } \\
\text { im Verhältnis zum Außendurch- } \\
\text { messer }\end{array}$ & $\begin{array}{l}\text { erhöhter Innendurchmesser im } \\
\text { Verhältnis zum Außendurchmesser }\end{array}$ \\
\hline kein Tumoreinwachsen & $\begin{array}{l}\text { Tumoreinwachsen möglich (nicht } \\
\text { gecovert) }\end{array}$ \\
\hline $\begin{array}{l}\text { mäßige Anpassung an die } \\
\text { anatomischen Gegebenheiten }\end{array}$ & $\begin{array}{l}\text { gute Anpassung an die anatomi- } \\
\text { schen Gegebenheiten }\end{array}$ \\
\hline $\begin{array}{l}\text { mukoziliäre Clearance stark } \\
\text { gestört }\end{array}$ & $\begin{array}{l}\text { mukoziliäre Clearance weniger stark } \\
\text { beeinträchtigt (nicht gecovert) }\end{array}$ \\
\hline
\end{tabular}

Es werden Metallstents mit vollständigem, teilweisem und ohne Kunststoffüberzug(coverung) unterschieden. Nicht gecoverte Stents haben gegenüber den gecoverten den Vorteil, dass die mukoziliäre Clearance teilweise erhalten bleibt. Fehlt der Kunststoffüberzug, kann der Tumor jedoch durch die Maschen wachsen und dadurch eine erneute Stenosierung verursachen [22,41]. Nicht gecoverte Stents werden daher nur noch selten implantiert. Vollständig gecoverte Stents sind im Bronchialsystem schlechter verankert und neigen daher häufiger zu Dislokation als nicht gecoverte oder teilweise gecoverte Stents [3]. Frakturen der Metallmaschen können vorkommen, sind jedoch selten [40].

Die Entfernung von Metallstents ist in der Regel möglich, jedoch kann das Einwachsen von Tumor- oder Granulationsgewebe durch nicht gecoverte Stentmaschen die Entfernung sehr erschweren oder unmöglich machen [40]. Bei allen Stentarten, auch bei gecoverten und bei Silikonstents, kann es durch Tumorwachstum oder Granulationsgewebsbildung zu einer Einengung im Bereich der Stentenden kommen. Ein Nachteil der Metall- stents ist der im Vergleich zum Silikonstent höhere Preis. Vorund Nachteile von Metall- und Silikonstents sind in $\bullet$ Tab. 2 zusammengefasst.

\section{Stenose durch Kombination von exophytischem Wachstum und Kompression}

Stenosen, die durch eine Kombination von exophytischem Wachstum und Kompression verursacht werden, sollten, falls nach Abtragung der exophytischen Anteile weiterhin eine relevante Reststenose besteht, durch additive Stentimplantation geweitet werden. Da Kombinationsstenosen häufig auftreten, sollte der Untersucher Techniken der Tumorabtragung und der Stentimplantation beherrschen.

\section{Schlussfolgerung}

Symptomatische Stenosen der Atemwege müssen nicht mehr fatalistisch hingenommen, sondern können häufig erfolgreich endoskopisch behandelt werden. Der Erfolg der Therapie ist dabei entscheidend von einer exakten Planung und der Auswahl der geeigneten Therapieverfahren abhängig.

\section{Interessenkonflikt}

\section{$\nabla$}

J. Hetzel hat Vortragshonorare von Erbe und Leufen medical erhalten. M. Böckeler gibt an, dass kein Interessenkonflikt besteht.

\section{Literatur}

1 Jemal A, Bray F, Center MM et al. Global cancer statistics. Cancer J Clin 2011; 61: 69

2 Gompelmann D, Eberhardt R, Herth FJ. Advanced malignant lung disease: what the specialist can offer. Respiration 2011; 82: 1111-1123

3 Freitag L. Airway stents. Eur Respir Mon 2010; 48: 190-217

4 Leland Oviatt P, Stather DR, Michaud G et al. MDCM Exercise Capacity, Lung Function, and Quality of Life After Interventional Bronchoscopy. J Thorac Oncol 2011; 6: 38-42

5 Hautmann H et al. Empfehlung zur Sedierung in der flexiblen Bronchoskopie. Pneumologie 2011; 65: 647-652

6 Vergnon J-M, Huber RM, Moghiss K. Place of cryotherapy, brachytherapy and photodynamic therapy in therapeutic bronchoscopy of lung cancers. Eur Respir J 2006; 28: 200 - 218

7 Homasson JP, Renault P, Angebault $M$ et al. Bronchoscopic cryotherapy for airway strictures caused by tumors. Chest 1986; 90: 159-164

8 Mathur PN, Wolf KM, Busk MF et al. Fiberoptic bronchoscopic cryotherapy in the management of tracheobronchial obstruction. Chest 1996; 110: $718-723$

9 Hetzel M, Hetzel J, Schumann $C$ et al. Cryorecanalization: a new approach for the immediate management of acute airway obstruction. Thorac Cardiovasc Surg 2004; 127: 1427-1431

10 Schumann C, Hetzel M, Babiak AJ et al. Endobronchial tumor debulking with a flexible cryoprobe for immediate treatment of malignant stenosis. J Thorac Cardiovasc Surg 2010; 139: 997-1000 [Epub 2009 Aug 27]

11 Franke KJ, Szyrach M, Nilius G et al. Experimental study on biopsy sampling using new flexible cryoprobes: influence of activation time, probe size, tissue consistency, and contact pressure of the probe on the size of the biopsy specimen. Lung 2009; 187: 253-259

12 Hetzel J, Hetzel M, Hasel C et al. Old meet modern. Respiration 2008; 76: $193-197$

13 Barlow DE. Endoscopic applications of electrosurgery: a review of basic principles. Gastrointest Endoscop 1982; 28: 73 - 76

14 Saenghirunvattana S, Buakham C, Masakul $N$ et al. Management of Endobronchial Cancer Using Bronchoscopic Electrocautery. J Med Assoc Thai 2006; 89: 459-461

15 Bolliger CT, Sutedja TG, Strausz J et al. Therapeutic bronchoscopy with immediate effect: laser, electrocautery, argon plasma coagulation and stents. Eur Respir J 2006; 27: 1258 - 1271 
16 Grund KE, Storek D, Farin G. Endoscopic argon plasma coagulation (APC) first clinical experiences in flexible endoscopy. Endosc Surg Allied Technol 1994; 2: $42-46$

17 Grund KE, Straub T, Farin G. New haemostatic techniques: argon plasma coagulation. Baillieres Best Pract. Res. Clin. Gastroenterol 1999; 13: $67-84$

18 Dumon JF, Meric B, Bourcereau J et al. Principles for safety in application of Nd: YAG laser in bronchology. Chest 1984; 86: 278-284

19 Dutau H, Breen DP. Endobronchial laser treatment: an essential tool in therapeutic bronchoscopy. Eur Respir Mon 2010; 48: 149-160

20 Dumon MC, Cavaliere S, Dumon JF. Endobronchial laser therapy. Rev Mal Respir 1999; 16: 601-608

21 Kvale PA, Eichenhorn MS, Radke JR et al. YAG laser photoresection of lesions obstructing the central airways. Chest 1985; 87: 283-288

22 Beamis JF, Becker HD, Cavaliere $S$ et al. ERS/ATS statement on interventional pulmonology. Chairmen: C. T. Bolliger, P. N. Mathur. Eur Respir J 2002; 19: 356-373

23 Allison R, Moghissi K, Downie G et al. Photodynamic therapy (PDT) for lung cancer. Photodiagnosis Photodyn Ther 2011; 8: 231 -239

24 Moghissi K, Dixon K. Is bronchoscopic photodynamic therapy a therapeutic option in lung cancer? Eur Respir J 2003; 22: 535-541

25 Dougherty TJ, Cooper MT, Mang TS. Cutaneous phototoxic occurrences in patients receiving Photofrin. Lasers Surg med 1990; 10: 485-488

26 Sutedja $G$, van Kralingen $K$, Schramel FM et al. Fibreoptic bronchoscopic electrosurgery under local anaesthesia for rapid palliation in patients with central airway malignancies: a preliminary report. Thorax 1994; 49: $1243-1246$

27 Reddy C, Majid A, Michaud G et al. Gas embolism following bronchoscopic argon plasma coagulation: a case series. Chest. 2008; 134: 1066 1069

28 Tellides G, Ugurlu B, Kim R et al. Pathogenesis of systemic air embolism during bronchoscopic Nd:YAG laser operations. Ann Thorac Surg 1998 65: $930-934$

29 Trendelenburg $F$. Beiträge zu den Operationen an den Luftwegen. Langenbecks Arch Chir 1872; 13: 335
30 Casal RF. Update in airway stents. Curr Opin Pulm Med 2010; 16: 321 328

$31 \mathrm{Hu} H-\mathrm{C}, \mathrm{Liu} \mathrm{Y}-\mathrm{H}, \mathrm{Wu} \mathrm{Y}-\mathrm{C}$ et al. Granulation tissue formation following Dumon airway stenting: the influence of stent diameter. Thorac Cardiovasc Surg 2011; 59: 163 - 168

32 Simoff M, Stermann DH, Ernst A. Obstruction of the central airways: evaluation and management. Lunn W ed. Thoracic endoscopy. Malden, Massachusetts, USA: Blackwell Futura; 2006

33 Dutau H, Toutblanc B, Lamb C et al. Use of the Dumon Y-stent in the management of malignant disease involving the carina: a retrospective review of 86 patients. Chest 2004 ; 126: 951 -958

34 Dumon JF. A dedicated tracheobronchial stent. Chest 1990; 97: 328 332

35 Dumon JF, Cavaliere S, Diaz-Jimenez JP. Seven-year experience with the Dumon prosthesis. J Bronchol 1996; 31: 6-10

36 Miyazawa T, Yamakido M, Ikeda S et al. Implantation of ultraflex nitinol stents in malignant tracheobronchial stenoses. Chest 2000; 118: 959 965

37 Herth F, Becker HD, LoCicero J et al. Successful bronchoscopic placement of tracheobronchial stents without fluoroscopy. Chest 2001; 119: $1910-1912$

38 Breitenbucher A, Chhajed PN, Brutsche MH et al. Long-term follow-up and survival after Ultraflex stent insertion in the management of complex malignant airway stenoses. Respiration 2008; 75: 443-449

39 Fruchter $O$, Raviv Y, Fox BD et al. Removal of metallic tracheobronchial stents in lung transplantation with flexible bronchoscopy. Journal of Cardiothoracic Surgery 2010; 5: 72

40 Herth F, Eberhardt $R$. Endobronchiales Stenting. Kardiovaskuläre Medizin 2007; 10: 101 - 107

41 Monnier P, Mudry A, Stanzel F et al. The use of the covered Wallstent for the palliative treatment of inoperable tracheobronchial cancers. A prospective, multicenter study. Chest 1996; 110: $1161-1168$

42 Folch E, Mehta AC. Airway interventions in the tracheobronchial tree. Semin Respir Crit Care Med 2008; 29: 441 - 452 